\title{
Preparation of Cellulose Nanofiber-reinforced Gelatin Hydrogel and Optimization for 3D Printing Applications
}

\author{
Yani Jiang, ${ }^{\mathrm{a}}$ Xiaodong Xv, ${ }^{\mathrm{a}}$ Dongfang Liu, ${ }^{\mathrm{b}}$ Zhe Yang, ${ }^{\mathrm{a}}$ Qi Zhang ${ }^{\mathrm{b}}$, Hongcan Shi, ${ }^{\mathrm{c}}$ Guoqi \\ Zhao, ${ }^{\mathrm{a}}$ Jiping Zhou ${ }^{\mathrm{b}, *}$
}

\begin{abstract}
Gelatin (GEL) obtained from animals is famous for its biocompatibility and biodegradability. However, its poor mechanical properties limits possible applications as bio-inks to fabricate tissue scaffolds through threedimensional (3D) printing. In this work, a high strength hydrogel based on cellulose nanofibers and GEL (CNF/GEL) was designed for 3D printing. Scanning electron microscopy and breaking strength results indicated that a CNF filling content of $10 \%$ was the best content in the CNF/GEL hydrogels. The rheological properties of the samples with different solid contents were investigated, and the 10\%-CNF/GEL-5 hydrogel was proposed for 3D printing. Then, a printing strategy with optimal conditions, including a crosslinking procedure for obtaining a 3D scaffold, was proposed. The biocompatibility of G-10\%-CNF/GEL-5 was also investigated using CCK-8 and Hoechst 33342/PI double-staining assays. These results confirmed that the 10\%-CNF/GEL-5 composite hydrogel has potential to be used as a 3D bio-ink for application in tissue repair.
\end{abstract}

Keywords: 3D printing; Cellulose nanofibers/Gelatin (CNF/GEL); Hydrogels; Scaffold

Contact information: a: College of Animal Science and Technology, Yangzhou University, No. 48 East Wenhui Road, Yangzhou, China; b: College of Mechanical Engineering, Yangzhou University, No. 196 West Huayang Road, Yangzhou, China; c: Medical college, Yangzhou University, No. 11 Huaihai Road, Yangzhou, China; *Corresponding author: Jiping Zhou (E-mail: jpzhou@yzu.edu.cn)

\section{INTRODUCTION}

Three-dimensional (3D) printing, also known as additive manufacturing, can be used to fabricate modular and patient-specific scaffolds with a high structural complexity and design flexibility. This technique makes it possible to design and manufacture tissue structures based on common medical imaging, such as computed tomography and magnetic resonance imaging, which cannot be accomplished with conventional fabrication techniques. Currently, 3D printing is used in many areas, such as craniofacial implants (Pereira and Bártolo 2015), dental molds, crowns and implants (Dawood et al. 2015; Obregon et al. 2015), prosthetic parts (Wong 2015), on-demand medical equipment (Jacobs et al. 2008), surgical models (Park and Gerecht 2015), tissue models for drug discovery (Do et al. 2015), and scaffolds for the regeneration of tissue, including skin and bone (Singh et al. 2016). Three-dimensional printing has many special advantages, such as the capability of fabricating patient-specific devices, controlling orientation and porosity, and combining multiple materials, which has attracted the attention of many researchers and enabled the development of many breakthrough treatments and devices. Generally, 3D printing inks must meet the following requirements: (1) printable; (2) biocompatible; (3) appropriate mechanical properties; (4) good degradation kinetics; (5) safe degradation byproducts; and (6) tissue biomimicry (Zheng et al. 2016). 
Natural hydrogels, including alginate (Colosi et al. 2016), gelatin (GEL) (Bhattacharjee et al. 2015), collagen (COL) (Skardal et al. 2012), fibrin (Skardal et al. 2012), hyaluronate acid (Highley et al. 2015), chitosan, and agarose (Müller et al. 2015), possess many unique properties, such as biocompatibility, biodegradability, and biomimicry of soft tissue. Thus, these materials have attracted attention as possible bioinks for the preparation of implantable scaffolds in the tissue engineering (TE) field. GEL is a natural polymer obtained by hydrolysis from COL, which is the major protein component of bone, cartilage, skin, and connective tissue and the major constituent of all extracellular matrices (ECM) in animals (Zheng et al. 2013).

Because of its biocompatibility and biodegradability, GEL is commonly used in pharmaceutical and medical applications (Lu et al. 2013). With respect to COL, GEL does not express antigenicity and is completely resorbable in vivo; furthermore, it is much cheaper and easier to obtain GEL in concentrated solutions (Lu et al. 2013). The properties and mechanism of gelation have been widely studied (Gao et al. 2013; Kim et al. 2013; Lequeux et al. 2015). However, its poor mechanical properties limit its possible applications as a biomaterial.

Recently, nanocelluloses, including cellulose nanofibrils (CNF) and cellulose nanocrystals (CNC), isolated from natural plants have been considered for biomedical applications because they present low toxicity, biocompatibility, and excellent mechanical properties, which make them excellent candidates as reinforcement materials for improving the mechanical properties of biomaterials (Hassan et al. 2012). Cellulose has been used to prepare scaffolds for tissue-engineered meniscus and blood vessel repair (Bäckdahl 2008). Furthermore, scaffolds prepared from bacterial cellulose/COL have also been confirmed to be biocompatible (Mathew et al. 2013). These studies confirmed that these fibrous networks, with excellent mechanical strengths, allow for human ligament cell and human endothelial cell adhesion, growth, and differentiation.

In this study, the authors focused on designing a hydrogel with CNF and GEL (CNF/GEL) and investigated its potential for 3D bioprinting applications. The CNF was isolated from Humulus japonicus stem (HJS, also called Japanese hop and regarded as an aggressive invader of forest edge habitats, floodplains, and riparian zones) by high-speed stirring combined with ultrasound. The morphological characteristics of the CNF and CNF/GEL hydrogel samples were separately observed with scanning electron microscopy (SEM) and transmission electron microscopy (TEM).

A suitable hydrogel was proposed for $3 \mathrm{D}$ printing by examining the mechanical properties of the CNF/GEL composite hydrogels with different CNF filling contents (3\%, $5 \%, 10 \%, 15 \%$, and 20\%) and the rheological properties of the CNF/GEL composite hydrogels with different solid contents. The temperatures of the barrel and working stage during the 3D printing process were investigated and optimized. Additionally, the crosslinking ability of the printed scaffold using genipin, which is a material obtained from the fruit of Gardenia jasminoides (Dimida et al. 2015) and a natural cross-linking agent, was determined by 2,4,6-trinitrobenzene sulfonic acid (TNBS) assay.

The chemical structures of the samples were investigated with Fourier transform infrared (FTIR) spectroscopy. Finally, the cytotoxicity and biocompatibility of G-10\%CNF/GEL-5 were evaluated using Cell Counting Kit 8 (CCK-8) and Hoechst 33342/PI double-staining assays. 


\section{EXPERIMENTAL}

\section{Materials}

The HJS was harvested from Jiangsu Province, China. The GEL was of bioreagent grade and purchased from Macklin Biochemical Reagent Co., Ltd. (Shanghai, China). The genipin was obtained from Linchuan Zhixin Biotechnology Co., Ltd (Fuzhou, Jiangxi). All of the other chemicals were of analytical reagent grade and supplied by Sinopharm Chemical Reagent Co., Ltd. (Shanghai, China).

\section{Methods}

\section{Preparation of the $\mathrm{CNF}$}

The preparation of bleached HJS material isolated from the HJS samples was reported in our previous study (Jiang et al. 2017). Briefly, the HJS powder was treated with $4 \%$ sodium hydroxide solution $\left(W_{\mathrm{HJS}} / V_{\mathrm{NaOH}}=1 / 15\right)$ at $80{ }^{\circ} \mathrm{C}$ for $2 \mathrm{~h}$ and then treated 3 times with $5 \%$ acidified sodium chlorite $\left(W_{\mathrm{HJS}} / V_{\mathrm{ASC}}=1 / 15\right)$ at $80{ }^{\circ} \mathrm{C}$ for $6 \mathrm{~h}(2 \mathrm{~h}$ each time $)$ to remove hemicellulose and lignin. Next, a given mass of the bleached HJS suspension (1 wt.\%) was treated using high-speed agitation (20000 rpm) for $10 \mathrm{~min}$, which was followed by high-power ultrasonication $(1200 \mathrm{~W})$ for $15 \mathrm{~min}$ to isolate the nanofibers. The ultrasonic treatment was performed in an ice bath; the ice was maintained throughout the entire ultrasonication process.

\section{Preparation of the CNF/GEL hydrogels}

A certain amount of GEL was added to a phosphate-buffered solution (PBS) and stirred at $40{ }^{\circ} \mathrm{C}$ until the GEL was completely dissolved. Different quantities of CNF solution (1 wt.\%) were separately added to the GEL solution and stirred evenly at $40{ }^{\circ} \mathrm{C}$ for $1 \mathrm{~h}$. To eliminate bubbles, these solutions were subjected to a vacuum-pumping treatment. These prepared hydrogel samples had the same GEL content of 5\% $\left(\mathrm{GEL} \%=W_{\text {SolidGEL }} / W_{\text {Solution }} * 100 \%\right)$ and different $\mathrm{CNF}$ filling contents $\left(\mathrm{CNF} \%=W_{\text {SolidCNF}} /\right.$ $\left.W_{\text {Solid GEL }}{ }^{*} 100 \%\right)$ of $0 \%, 3 \%, 5 \%, 10 \%, 15 \%$, and $20 \%$. These hydrogel samples are referred to as GEL-5, 3\%-CNF/GEL-5, 5\%-CNF/GEL-5, 10\%-CNF/GEL-5, 15\%CNF/GEL-5, and 20\%-CNF/GEL-5, respectively. These hydrogel samples and their compositions are listed in Table 1.

Table 1. Chemical Composition of the Hydrogel Samples

\begin{tabular}{|c|c|c|c|c|c|}
\hline Sample & $\begin{array}{c}\text { GEL Content } \\
(\%)\end{array}$ & $\begin{array}{c}\text { GEL } \\
\text { Concentration } \\
\left(\mathrm{mg}^{-1} \mathrm{~mL}^{-1}\right.\end{array}$ & $\begin{array}{c}\text { CNF Filling } \\
\text { Content } \\
(\%)\end{array}$ & $\begin{array}{c}\text { CNF } \\
\text { Concentration } \\
\left(\mathrm{mg}^{-1} \mathrm{~mL}^{-1}\right)\end{array}$ & $\begin{array}{c}\text { Total Solids } \\
\text { Concentration } \\
\left(\mathrm{mg}^{\left.-\mathrm{mL}^{-1}\right)}\right.\end{array}$ \\
\hline GEL-5 & 5 & 50 & 0 & 0 & 50 \\
\hline $3 \%-C N F / G E L-5$ & 5 & 50 & 3 & 1.5 & 51.5 \\
\hline $5 \%-C N F / G E L-5$ & 5 & 50 & 5 & 2.5 & 52.5 \\
\hline $10 \%-C N F / G E L-5$ & 5 & 50 & 10 & 5 & 55 \\
\hline $15 \%-C N F / G E L-5$ & 5 & 50 & 15 & 7.5 & 57.5 \\
\hline $20 \%-C N F / G E L-5$ & 5 & 50 & 20 & 10 & 60 \\
\hline $10 \%-C N F / G E L-3$ & 3 & 30 & 10 & 3 & 33 \\
\hline $10 \%-C N F / G E L-7$ & 7 & 70 & 10 & 7 & 77 \\
\hline
\end{tabular}




\section{Characterization of the morphological features}

The morphology of the CNF was determined using TEM (Tecnai 12, Philips Co., Ltd., Amsterdam, Holland). A drop of diluted suspension was deposited on the surface of a carbon-coated copper grid and then negatively stained with phosphotungstic acid for 3 min. The images were captured at an accelerating voltage of $120 \mathrm{kV}$.

The morphology of the alkali-treated HJS fibers, bleached HJS fibers, and hydrogel samples was characterized using SEM (S-4800, Hitachi Co., Ltd., Tokyo, Japan). The samples were sputtered with gold, and the images were captured at an accelerating voltage of $15 \mathrm{kV}$.

The diameter and length of the CNF were measured for approximately 50 representative $\mathrm{CNF}$ using a digital image-processing program (ImageJ, National Institutes of Health, USA), and the average values are reported in the ensuing sections.

\section{Mechanical property measurements}

The mechanical properties of the hydrogel samples were measured via a uniaxial compression test method, which was proposed by Zheng et al. (2016), using a universal tensile testing machine (HLD-0824, Handpi Co., Ltd., Leqing, China). The hydrogel samples were formed into cylinders with a diameter of $15 \mathrm{~mm}$ and height of $10 \mathrm{~mm}$. The test was performed at a compression speed of $0.5 \mathrm{~mm} . \mathrm{s}^{-1}$ at room temperature $\left(15^{\circ} \mathrm{C}\right)$. Five tests were performed on each sample and the average values are reported in the ensuing sections.

\section{Rheological characterization}

The rheological characterizations of the samples with different solid contents were investigated using a discovery hybrid rheometer (DHR-3, TA instruments, New Castle, DE, USA). These samples are referred to as $10 \%$-CNF/GEL-3, $10 \%$-CNF/GEL-5, and 10\%CNF/GEL-7. These samples and their compositions are listed in Table 1.

First, the strain amplitudes of all of the hydrogel samples were verified to ensure that the measurements were conducted within the linear viscoelastic region (LVR). A temperature sweeping test was performed to observe the sol-gel transformation temperature ( $\left.T_{\text {sol-gel }}\right)$ of GEL-5, 10\%-CNF/GEL-3, $10 \%$-CNF/GEL-5, and 10\%-CNF/GEL7. The hydrogel samples were analyzed by employing a temperature heating rate of $5{ }^{\circ} \mathrm{C} \cdot \mathrm{min}^{-1}$ from $3{ }^{\circ} \mathrm{C}$ to $50{ }^{\circ} \mathrm{C}$ in the oscillation mode using a strain of $1 \%$ and frequency of $1 \mathrm{~Hz}$.

Next, to investigate the effect of the shear rate, the viscosities of $10 \%$-CNF/GEL$3,10 \%-\mathrm{CNF} / \mathrm{GEL}-5$, and $10 \%$-CNF/GEL-7 were measured at shear rates ranging from 0.1 to $100 \mathrm{~s}^{-1}$ at a constant temperature of $10^{\circ} \mathrm{C}$.

\section{$3 D$ printing}

A 3D bioprinter (Bio-Architect, Regenovo Biotechnology Co. Ltd., Hangzhou, China) was used to print the 10\%-CNF/GEL-5 hydrogel. Scaffolds were designed and inputted into the printer software. The printing process was conducted using an extrusionbased printing nozzle, and the hydrogel flow was controlled by a pressure regulator. The 10\%-CNF/GEL-5 hydrogel was loaded into a barrel. The temperatures of the barrel and work stage were precisely controlled through the water circulation cooling system of the printer. The printability of the hydrogel was assessed using a constant nozzle diameter of $210 \mu \mathrm{m}$ with the combination of a printing pressure of $0.28 \mathrm{MPa}$ and printing speed of 10 $\mathrm{mm} . \mathrm{s}^{-1}$ at different barrel $\left(10^{\circ} \mathrm{C}, 15^{\circ} \mathrm{C}, 20^{\circ} \mathrm{C}\right.$, and $\left.25^{\circ} \mathrm{C}\right)$ and work stage $\left(10{ }^{\circ} \mathrm{C}, 17^{\circ} \mathrm{C}\right.$, 
and $\left.25^{\circ} \mathrm{C}\right)$ temperatures. A mesh structure $\left(0^{\circ} / 90^{\circ}\right.$ strut structure $)$ was obtained using layer-by-layer deposition. Specifically, the first layer was generated by dispensing the hydrogel onto a metal slide placed on the work stage, and the subsequent layers were constructed by the deposition of alternating horizontal and vertical hydrogel filaments in a bottom-up building pattern.

The 3D printed constructs were observed using a digital camera (S2900HD, FinePix Co. Ltd., Chiba, Japan). The pore sizes of the printed scaffolds were measured using a digital image-processing program (ImageJ, National Institutes of Health, Bethesda, MD, USA). The results are given as the average of five samples.

\section{Crosslinking efficiency}

After 3D printing, the scaffolds were separately soaked in PBS ( $\mathrm{pH}=7.41)$ with genipin concentrations of $0.5 \mathrm{mM}, 2 \mathrm{mM}$, and $3.5 \mathrm{mM}$ at room temperature for $6 \mathrm{~h}, 12 \mathrm{~h}$, $24 \mathrm{~h}$, and $48 \mathrm{~h}$. Then, the crosslinked structures were repeatedly washed with triple-distilled water. The color changes of the printed constructs at different crosslinking times were observed using a digital camera (S2900HD, FinePix Co. Ltd., Chiba, Japan).

The crosslinking efficiency of the genipin with GEL was determined by comparing the lysine content before and after crosslinking using the TNBS assay (Ofner and Bubnis 1996). The $\varepsilon$-amino groups of the lysine in the GEL and/or unreacted GEL, which did not crosslink with genipin, can react with TNBS to form a soluble complex. After a freezedrying treatment, printed scaffolds with the same mass $(10 \mathrm{mg})$ were separately immersed in $2 \mathrm{~mL}$ of a solution containing $1 \mathrm{~mL}$ of $4 \%(\mathrm{w} / \mathrm{v})$ di-sodium hydrogen or thophosphate and $1 \mathrm{~mL}$ of $0.5 \%(\mathrm{v} / \mathrm{v})$ TNBS reagent, and they were incubated at $40{ }^{\circ} \mathrm{C}$ for $3 \mathrm{~h}$. Then, 3 $\mathrm{mL}$ of $\mathrm{HCl}\left(6 \mathrm{~mol} \cdot \mathrm{L}^{-1}\right)$ were added to the mixture to terminate the reaction, and the mixture was incubated at $60^{\circ} \mathrm{C}$ for $2 \mathrm{~h}$. After an appropriate dilution, the absorbance of the solution was measured at $345 \mathrm{~nm}$ using an ultraviolet-visible spectrophotometer (7315Jenway, Jenway Co. Ltd., Staffordshire, UK). The crosslinking efficiency (\%) was calculated with Eq. 1:

$$
\% \text { Crosslinking }=\left[\left(A_{\text {uncrosslinkedscaffold }}-A_{\text {crosslinkedscaffold }}\right) / A_{\text {uncrosslinkedscaffold }}\right] \times 100 \%
$$

where $A_{\text {uncrosslinked scaffold }}$ is the absorbance of the printed scaffold without a crosslinking treatment and $A_{\text {crosslinked scaffold }}$ is the absorbance of the printed scaffold after crosslinking treatment. Tests were performed in triplicate; the average values were reported.

\section{In vitro cytotoxicity and cell morphology}

The G-10\%-CNF/GEL-5 hydrogel was immersed in Dulbecco's modified eagle's medium (DMEM) with fetal bovine serum (FBS, 10\%) and pen/strep (1\%) for $48 \mathrm{~h}$. Then, the mixture was centrifuged to collect the leaching liquor.

The CCK-8 assay (Dojindo, Shanghai, China) was used to investigate the cell growth inhibition according to the instructions given by the manufacturer. The leaching liquor was used as a growth medium for culturing $3 \mathrm{~T} 3$ cells. The cells were seeded in 96well plates at $1.0 \times 10^{3}$ cells per well with $100 \mu \mathrm{L}$ of leaching liquor followed by cultivation in an incubator $\left(37^{\circ} \mathrm{C}, 5 \% \mathrm{CO}_{2}\right)$ for $1 \mathrm{~d}, 3 \mathrm{~d}, 5 \mathrm{~d}$, and $7 \mathrm{~d}$. Five independent samples were used for each time interval and experimental group. Next, the CCK-8 reagent $(10 \mu \mathrm{L}$ per well) was added to the plate, and the 3T3 cells were cultured for another $2 \mathrm{~h}$ under the same conditions. A microplate reader (Thermo Scientific, Shanghai, China) was used to measure the absorbance at a wavelength of $450 \mathrm{~nm}$ to determine the number of proliferating cells. 
A Hoechst 33342/PI double-staining assay was used to examine the induction of apoptosis by the G-10\%-CNF/GEL-5 hydrogel. In this experiment, an Apoptosis and Necrosis Assay Kit (Beyotime Institute of Biotechnology, Shanghai, China) was used to evaluate the cell morphology. The 3 T3 cells were cultured in 24-well plates using the leaching liquor as a growth medium for $48 \mathrm{~h}$. After $48 \mathrm{~h}$, the $3 \mathrm{~T} 3$ cells were washed twice with PBS $(\mathrm{pH}=7.4)$ and then stained with Hoechst $33342\left(10 \mathrm{ng} \cdot \mathrm{mL}^{-1}\right)$ and PI $\left(10 \mathrm{ng} \cdot \mathrm{mL}^{-}\right.$

${ }^{1}$ ) for $20 \mathrm{~min}$ at $4{ }^{\circ} \mathrm{C}$. The condensed or fragmented nuclei of the apoptotic cells were observed with fluorescence microscopy (DMi8-M, Leica Co. Ltd., Solms, Germany), and the numbers of live and dead cells were counted with a digital image-processing program (ImageJ, National Institutes of Health, Bethesda, USA). The cells that were cultured in DMEM with $10 \%$ FBS at the same conditions acted as the control groups.

\section{Test standards}

The data were expressed as the means of at least three replicates plus or minus the standard deviation (SD). The results were analyzed with an analysis of variance. Least significant difference multiple comparison tests were used to determine the significant differences between the mean values. Significant differences between two groups were determined using two-tailed Student's t-tests. A confidence level of 95\% $(p<0.05)$ was used, and the analyses were performed using SPSS software (Version 17.0, SPSS, Inc., Chicago, IL, USA).

\section{RESULTS AND DISCUSSION}

\section{Morphology and Microstructure of the Hydrogel Samples}

A photograph of the HJS is shown in Fig. 1a. The photographs and SEM images of the alkali-treated HJS and bleached HJS fibers are shown in Fig. 1b and 1c. The color of the HJS fibers changed from green to brown after the alkali treatment (Fig. 2b) and then white after bleaching (Fig. 2c). The variations of the color along with the chemical treatments were due to the chromogenic reactions of functional groups. A TEM image of the CNF is shown in Fig. 1d. The diameter of the CNF ranged from 9.74 to $42.21 \mathrm{~nm}$, and its mean value was $24.63 \pm 1.16 \mathrm{~nm}(p<0.05)$, while the length of the CNF ranged from 0.62 to $4.86 \mu \mathrm{m}$, and its mean value was $2.75 \pm 0.11 \mu \mathrm{m}(p<0.05)$. It was observed that the diameter of the CNF decreased significantly compared with the bleached HJS fibers. This occurred because the high-speed rotating water flow during the process of nanocrystallization produced strong impact and shear forces on the bleached HJS fibers, which caused strong mechanical collisions at a high frequency in the adjacent bleached HJS fibers and resulted in damages to the internal cell walls of the fibers.

The microstructures of the GEL-5, 3\%-CNF/GEL-5, 5\%-CNF/GEL-5, 10\%CNF/GEL-5, 15\%-CNF/GEL-5, and 20\%-CNF/GEL-5 hydrogel samples after lyophilization are shown in Fig. 1e and 1f, $1 \mathrm{~g}$ and $1 \mathrm{~h}, 1 \mathrm{i}$ and $1 \mathrm{j}, 1 \mathrm{k}$ and $11,1 \mathrm{~m}$ and $1 \mathrm{n}$, and 10 and 1p, respectively. The GEL had a very loose structure with large pores (Fig. 1e) and smooth-aperture walls, which can be clearly observed in Fig. 1f. Furthermore, the pores appeared to decease in size and the aperture walls appeared to become rougher with an increasing $\mathrm{CNF}$ filling content, which is shown in Fig. 1g to $1 \mathrm{p}$. 

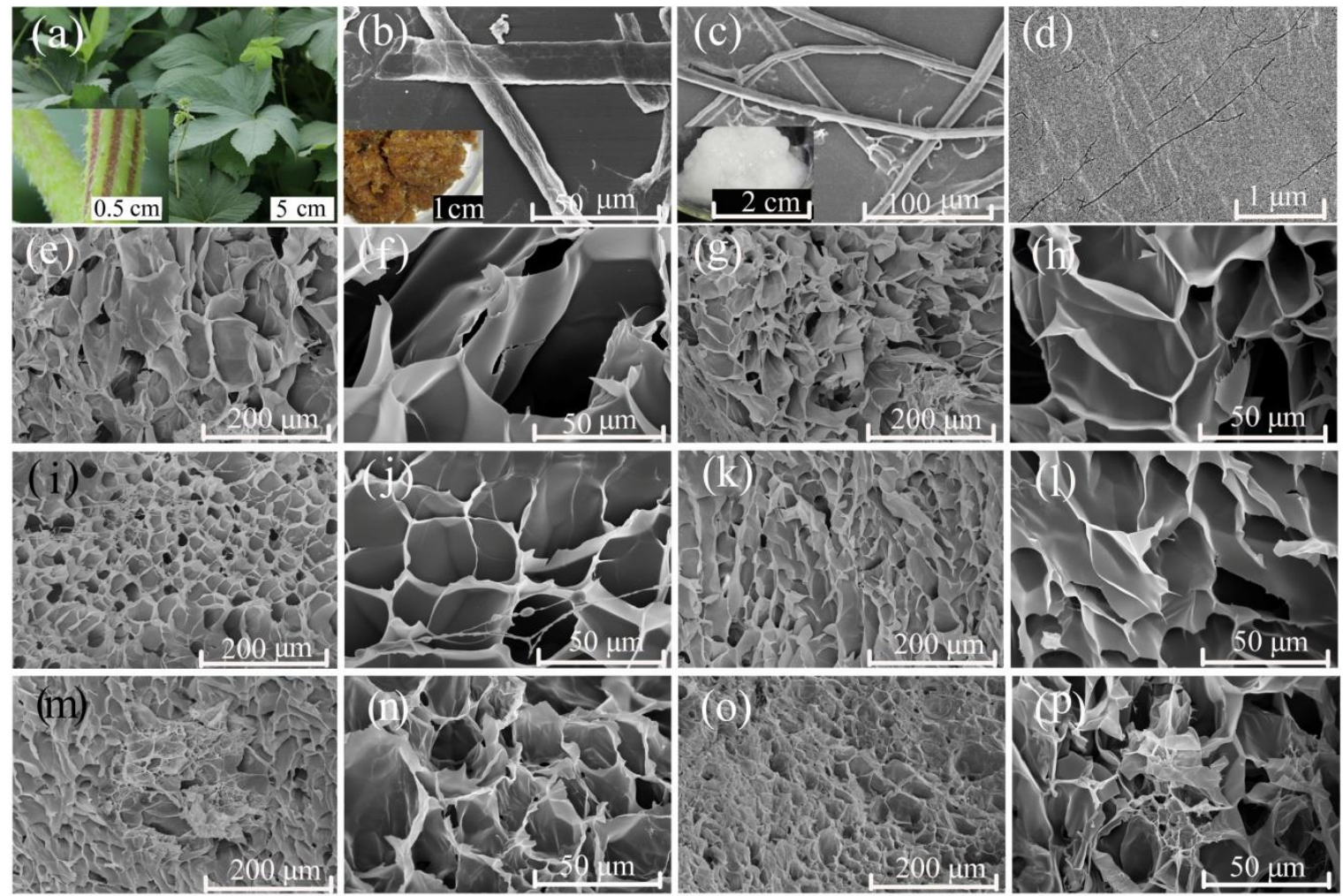

Fig. 1. Photograph of the crude HJS (a); Photographs and SEM images showed variations in color and fiber diameter for HJS after alkali treatment (b) followed by bleaching (c); TEM image of the CNF fibers (d) showed a significant diameter decrease compared with the bleached HJS fibers; (d); SEM images of GEL-5 (e, f), 3\%-CNF/GEL-5 (g, h), 5\%-CNF/GEL-5 (i, j), 10\%-CNF/GEL-5 (k, l), $15 \%-C N F / G E L-5(m, n)$, and $20 \%-C N F / G E L-5(o, p)$. With an increasing CNF filling content, the pores in the hydrogel samples appeared to decease in size, and the aperture walls became rougher and rougher.

Before lyophilization, all of the hydrogel samples were stored at $-20{ }^{\circ} \mathrm{C}$ for $24 \mathrm{~h}$, which caused the water in the hydrogels to gradually form crystal nuclei and ice. In the process of lyophilization, the ice crystals directly sublimated from the hydrogel samples under vacuum, which resulted in porous structures. The sizes of the pores reflected the scale of the original ice crystals (Wang et al. 2017). With an increasing CNF filling content, the hydrogel structure became stronger. Therefore, higher CNF filling contents caused the formation of smaller ice crystals, which resulted in gradually decreasing pore sizes and an increasing structural compactness. This is shown in Fig. $1 \mathrm{~g}$ to $1 \mathrm{p}$.

\section{Mechanical Property Measurements}

Compression tests were performed to evaluate the mechanical strength of the hydrogel samples. The compressive breaking strength of the GEL-5, 3\%-CNF/GEL-5, 5\%CNF/GEL-5, 10\%-CNF/GEL-5, 15\%-CNF/GEL-5, and 20\%-CNF/GEL-5 hydrogel samples is displayed in Fig. 2a. When the GEL content was kept constant at 5\% and the CNF filling content increased $(3 \%, 5 \%, 10 \%, 15 \%$, and 20\%), the breaking strengths of the composite hydrogel samples were all improved compared with the pure GEL hydrogel. The breaking strengths of GEL-5, 3\%-CNF/GEL-5, 5\%-CNF/GEL-5, 10\%-CNF/GEL-5, 15\%-CNF/GEL-5, and 20\%-CNF/GEL-5 were $43.32 \mathrm{KPa}, 89.01 \mathrm{KPa}, 154.68 \mathrm{KPa}, 249.37$ 
$\mathrm{KPa}, 172.47 \mathrm{KPa}$, and $102.71 \mathrm{KPa}$, respectively. Thus, the breaking strength increased at first and then decreased with an increasing $\mathrm{CNF}$ filling content. A maximum breaking strength of $249.37 \mathrm{KPa}$ was reached at a CNF filling content of $10 \%$, which was approximately 5.75-fold higher than that of the pure GEL, i.e., $43.32 \mathrm{KPa}$. The abundant hydroxyl groups existing in the CNF molecules could form intermolecular hydrogen bonds with the carboxyl or amino groups in the GEL macromolecules (Li et al. 2014). Additionally, the long chain of CNF can also interact with the GEL to form a complex triple-helix long chain structure (Peresin et al. 2010). Therefore, the appropriate addition of CNF could improve the mechanical properties and stability of the GEL. In addition, the strain for $10 \%$-CNF/GEL-5 reached a maximum of $66.21 \%$, which was significantly higher than that of the pure GEL-5 of 54.06\% as shown in Fig. 2b. This result was because that the addition of CNF could improve the crack resistance of GEL matrix resulting in a higher fracture toughness and shock resistance for the composite hydrogel. However, when the filling content of the CNF exceeded $10 \%$, the breaking strengths of the hydrogel samples began to decline. Most likely, in the 15\%-CNF/GEL-5 and 20\%-CNF/GEL-5 hydrogel samples (as shown in Fig. 1k to $1 \mathrm{n}$ ), the CNF aggregated because of its high specific surface area and surface activation energy, which reduced the ordered performance of the composite hydrogel structure and resulted in a reduction of the breaking strength. Therefore, the optimum CNF filling content was determined to be $10 \%$.
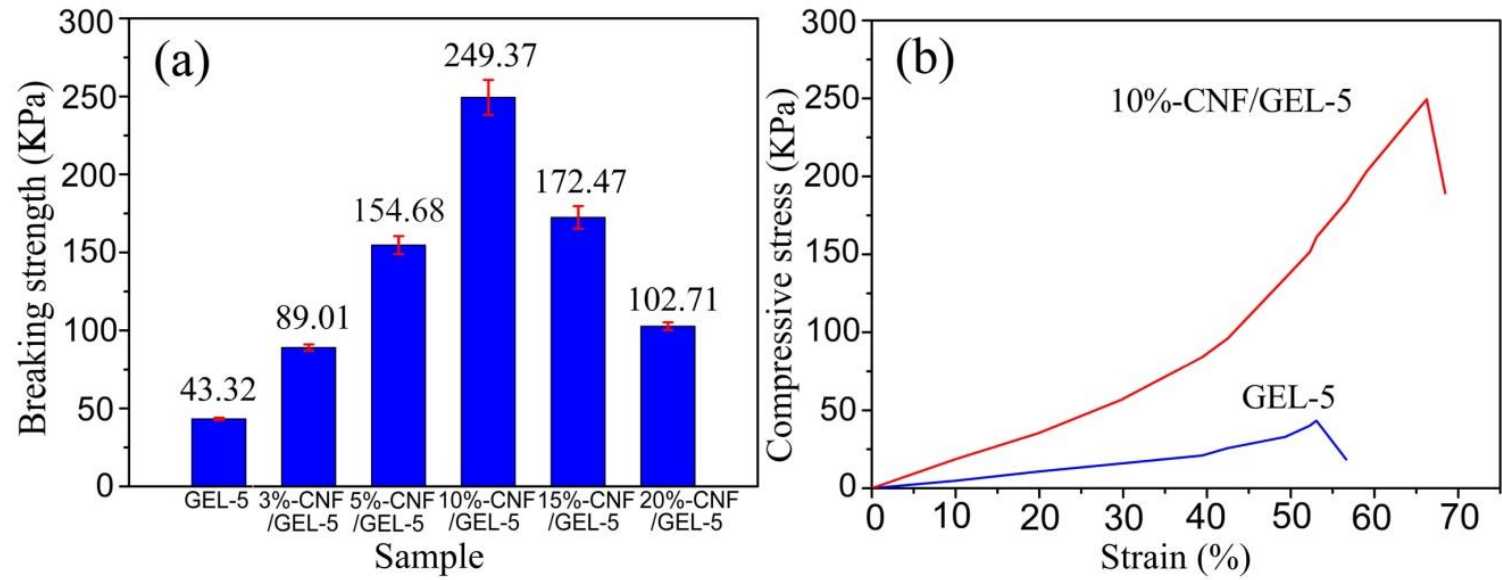

Fig. 2. Breaking strengths for the GEL-5, 3\%-CNF/GEL-5, 5\%-CNF/GEL-5, 10\%-CNF/GEL-5, $15 \%-C N F / G E L-5$ and $20 \%-C N F / G E L-5(p<0.05)(a)$. The breaking strength first showed a growth trend then began to decrease with an increasing CNF filling content, and reached a maximum at a CNF filling content of 10\%; Strain-stress curves of the GEL-5 and 10\%-CNF/GEL-5 scaffolds (b). The maximum deformation of 10-CNF/GEL-5 (66.21\%) was bigger than GEL-5 (54.06\%).

Qv (2013) prepared CNC/polyethylene glycol (PEG)/polylactic acid (PLA) ternary composite. Because of weak compatibility between the hydrophilic $\mathrm{CNC}$ and hydrophobic PLA, the optimal CNC content in the PEG/PLA matrix was only 2\%. Pei (2013) prepared CNC/GEL composite film with CNC content ranging from 3\% to $15 \%$. Their result suggested the mechanical strength of the composite reached a maximum when the CNC content in GEL was $9 \%$, which was approximate to our optimal value of $10 \%$. In addition, the $\mathrm{CNF}$ could be used as a reinforcer to improve the mechanical properties of other natural hydrogels, such as COL, fibrin, hyaluronate acid, alginate, chitosan, and agarose. 


\section{Rheological Properties}

In this part of the experiment, the GEL content was 3\%, 5\%, and $10 \%$ and the CNF filling content was kept constant at $10 \%$, which was confirmed to be the optimal filling amount for the GEL. Then, the effect of the solid contents on the rheological properties of the hydrogel samples was investigated. GEL is a thermo-sensitive polymer; thus, it is necessary to evaluate the rheological properties of the hydrogel samples at various temperatures. The storage $\left(G^{\prime}\right)$ and loss moduli $\left(G^{\prime \prime}\right)$ of GEL-5, 10\%-CNF/GEL-3, 10\%$\mathrm{CNF} / \mathrm{GEL}-5$, and 10\%-CNF/GEL-7 were investigated at temperatures ranging from $3{ }^{\circ} \mathrm{C}$ to $50{ }^{\circ} \mathrm{C}$ within the LVR. The $T_{\text {sol-gel }}$ occurs at the temperature at which $G$ ' intersects $G$ ', which indicates that the sample remains in the sol state if the ambient temperature is higher than the $T_{\text {sol-gel }}$ and converts to the gel state if the ambient temperature is lower than the $T_{\text {sol-gel. }}$ Figure 3 a shows that the $T_{\text {sol-gel was }} 23.6^{\circ} \mathrm{C}, 26.4^{\circ} \mathrm{C}, 30.1^{\circ} \mathrm{C}$, and $32.6^{\circ} \mathrm{C}$ for GEL5, 10\%-CNF/GEL-3, 10\%-CNF/GEL-5, and 10\%-CNF/GEL-7, respectively. This finding indicated that 10\%-CNF/GEL-3, 10\%-CNF/GEL-5, and 10\%-CNF/GEL-7 all exhibited thermo-sensitivity, similar to the pure GEL. Additionally, the $\underline{T}_{\text {sol-gel }}$ and $G$ ' both increased with an increasing solid contents of hydrogel. The increase in the $G$ ' indicated an improvement in the rigidity of the material, which was because of the increased degree of interaction between the CNF and GEL macromolecules in the composite hydrogel. As such, these results suggested that an increase in the solid content is favorable for improving the hydrogel rigidity.
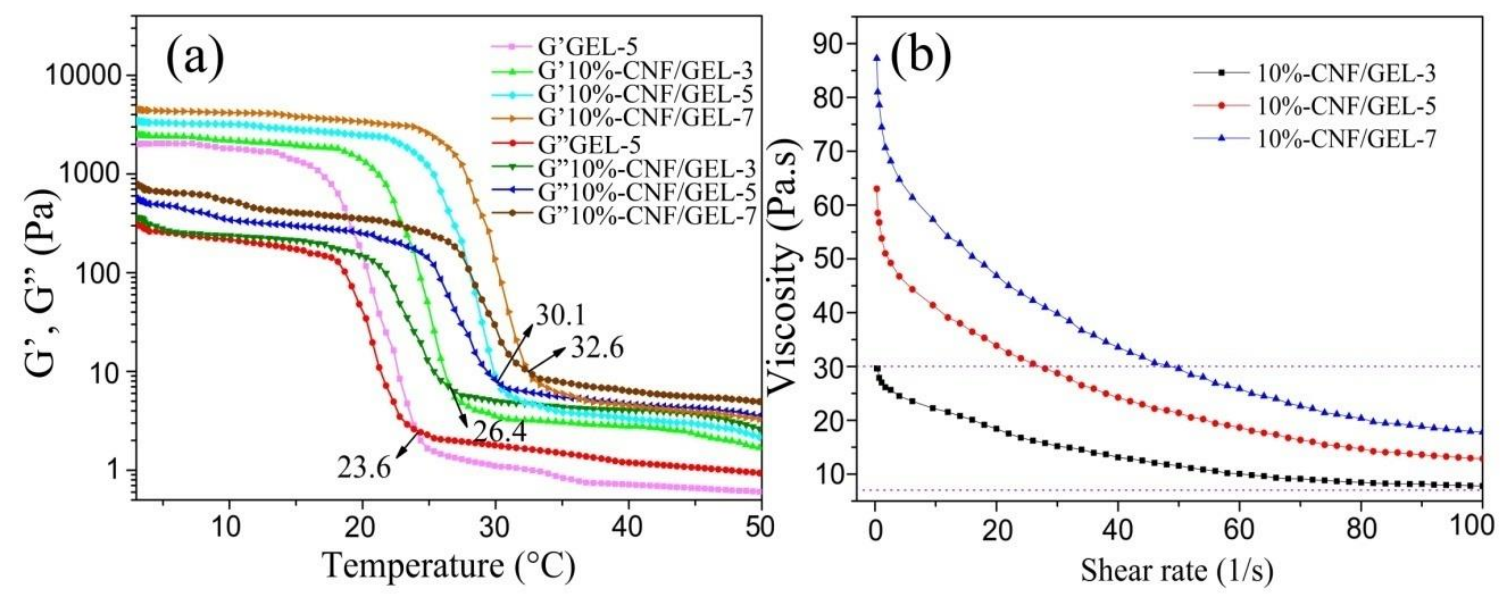

Fig. 3. Change in the storage modulus (G') and loss modulus (G") for the GEL-5, 10\%-CNF/GEL3, $10 \%-C N F / G E L-5$, and $10 \%-C N F / G E L-7$ hydrogels. It showed that $T_{\text {sol-gel }}$ and $G$ ' both increased with an increasing hydrogel solid contents (a); Viscosity of the 10\%-CNF/GEL-3, 10\%-CNF/GEL5 , and $10 \%-C N F / G E L-7$ hydrogels at different shear rates ranging from 0.1 to $100 \mathrm{~s}^{-1}$ at $10{ }^{\circ} \mathrm{C}$. The interval between the two gray dotted lines represented a suitable viscosity range ( $4 \sim 30 \mathrm{~Pa} . \mathrm{s})$ for $3 \mathrm{D}$ printing. The viscosity of $10 \%-\mathrm{CNF} / \mathrm{GEL}-7$ was out of the suitable viscosity range when the shear rates ranged from 20 to $60 \mathrm{~s}^{-1}$, which was an estimated shear rate range generated in the process of $3 \mathrm{D}$ printing.

Additionally, the rheological tests of the 10\%-CNF/GEL-3, 10\%-CNF/GEL-5, and $10 \%$-CNF/GEL-7 samples were performed at $10{ }^{\circ} \mathrm{C}$ to investigate how varying the shear rates affects the viscosity during the printing process and determine the suitability of these hydrogels as potential bio-inks. Ideally, the viscosity of a printable hydrogel should decrease so that it can flow through the nozzle of a 3D printer. Figure $3 \mathrm{~b}$ shows that the viscosity of all of the hydrogel samples decreased with an increasing shear rate. This 
phenomenon revealed that all of the hydrogel samples presented a clear shear-thinning behavior (a pseudo-plastic behavior), which was because of the temporary destruction of the physical interactions between the macromolecules and alignment of the polymer chains caused by a high shear rate. The shear rate generated in the process of 3D printing was estimated to range from approximately 20 to $60 \mathrm{~s}^{-1}$ ( $\mathrm{Ng}$ et al. 2016). Additionally, according to a previous report, the suitable viscosity for 3D printing ranges from 4 to $30 \mathrm{~Pa} \cdot \mathrm{s}$ (Das et al. 2015). Figure $3 \mathrm{~b}$ shows that the viscosity increased with an increasing hydrogel solid contents, and the viscosity of $10 \%$-CNF/GEL-7 was outside the ideal viscosity range when the shear rate ranged from $0 \mathrm{~Pa} \cdot \mathrm{s}$ to $100 \mathrm{~Pa} \cdot \mathrm{s}$. Together with the resulting values for the $G^{\prime}$, these findings prompted the authors to utilize 10\%-CNF/GEL-5 as a bio-ink in the subsequent $3 \mathrm{D}$ printing experiments because of its preferable rigidity and suitable viscosity.

\section{Hydrogel Printability}

Currently, there are two main methods for 3D printing. One is printing the hydrogel and cells separately, and the other is depositing a cell-laden hydrogel. As the former method can be used to more easily control the cell distribution in each printed layer $(\mathrm{Ng}$ et al. 2016), the authors focused on printing an acellular hydrogel through an extrusion-based technique. In general, the interconnectivity among the pores is a critical factor for determining the printability, which affects various cellular responses, such as the cell viability, growth, and differentiation (Murphy and Atala 2014). Because of this, the authors evaluated the printability of the $10 \%$-CNF/GEL- 5 hydrogel through the appearances of the pores in the printed constructs. In this experiment, a series of struts of a similar size were constructed at pneumatic pressures of $0.1 \mathrm{MPa}$ to $0.2 \mathrm{MPa}$. The strut height when the pore size was reduced to half $(200 \mu \mathrm{m})$ of the initially designed size $(400 \mu \mathrm{m})$ in the printing process was recorded as the final height, which can be used to assess the printability of the structure.

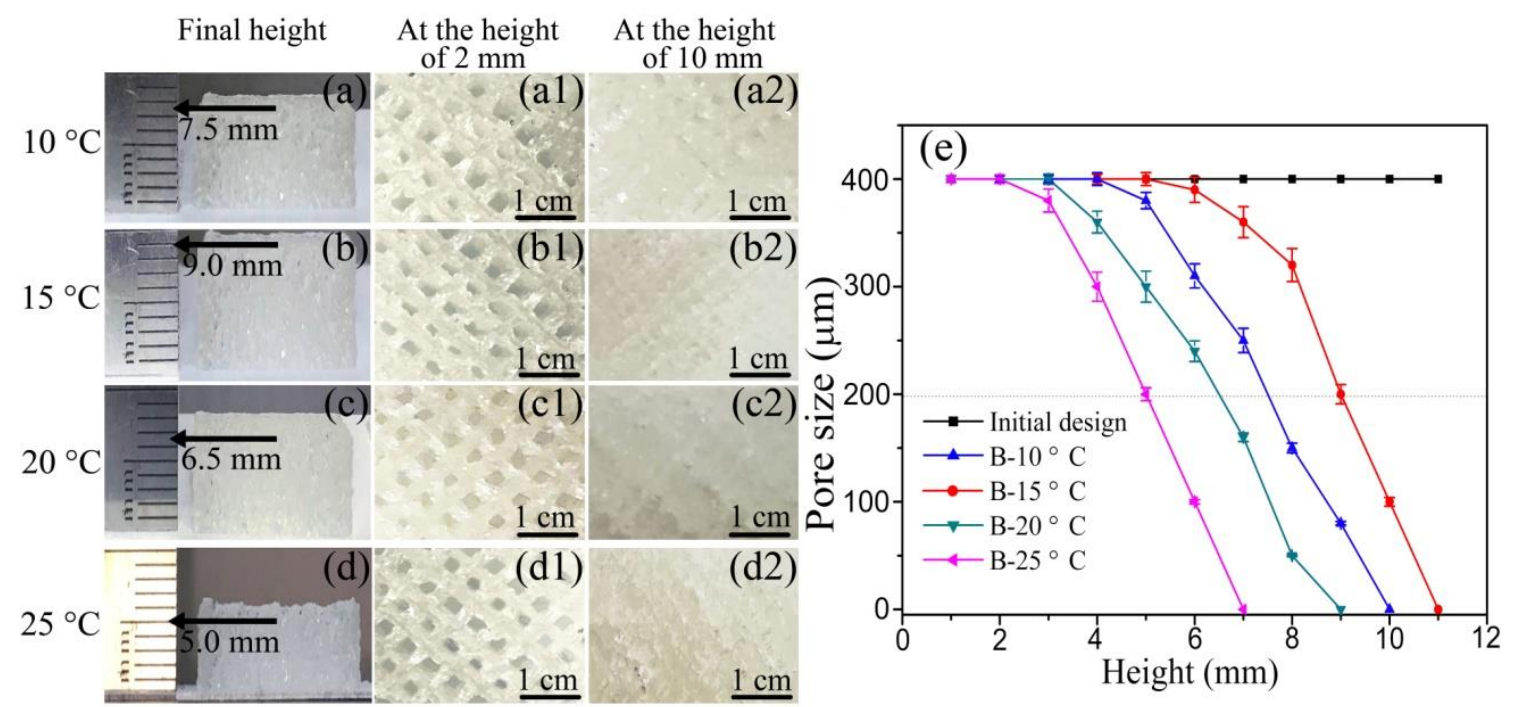

Fig. 4. Optical images of the struts printed at different barrel temperatures. The final heights for the struts printed at $\mathrm{B}-10^{\circ} \mathrm{C}(\mathrm{a}, \mathrm{a} 1, \mathrm{a} 2), \mathrm{B}-15^{\circ} \mathrm{C}(\mathrm{b}, \mathrm{b} 1, \mathrm{~b} 2), \mathrm{B}-20^{\circ} \mathrm{C}(\mathrm{c}, \mathrm{c} 1, \mathrm{c} 2)$, and $\mathrm{B}-25^{\circ} \mathrm{C}(\mathrm{d}, \mathrm{d} 1$, d2) were $7.5 \mathrm{~mm}, 9.0 \mathrm{~mm}, 6.5 \mathrm{~mm}$, and $5.0 \mathrm{~mm}$, respectively; The curves of the pore sizes with the increasing heights of the struts printed at different barrel temperatures $(p<0.05)$. The final strut height was the abscissa value of the intersection point of the curves and the gray dotted line at ordinate value of $200 \mu \mathrm{m}(\mathrm{e})$. 
First, $10 \%$-CNF/GEL-5 was printed at different barrel temperatures to evaluate the effect of the barrel temperature on the printability. Figure $4 a, 4 b, 4 c$, and $4 d$ show optical images of struts printed at four different barrel temperatures $\left(\mathrm{B}-10{ }^{\circ} \mathrm{C}, \mathrm{B}-15^{\circ} \mathrm{C}, \mathrm{B}-20{ }^{\circ} \mathrm{C}\right.$, and $\mathrm{B}-25^{\circ} \mathrm{C}$, respectively) and at the same work stage temperature of $17{ }^{\circ} \mathrm{C}$, and Fig. $4 \mathrm{e}$ shows the change in the pore size with the increasing strut height. The pores appeared to be intact when the strut height was $2 \mathrm{~mm}$, but they collapsed when the strut height was 10 $\mathrm{mm}$. The final heights for the struts printed at B-10 ${ }^{\circ} \mathrm{C}, \mathrm{B}-15{ }^{\circ} \mathrm{C}, \mathrm{B}-20{ }^{\circ} \mathrm{C}$, and $\mathrm{B}-25{ }^{\circ} \mathrm{C}$ were $7.5 \mathrm{~mm}, 9.0 \mathrm{~mm}, 6.5 \mathrm{~mm}$, and $5.0 \mathrm{~mm}$, respectively. Furthermore, it was observed in Fig. 4e that the pores processed at $25^{\circ} \mathrm{C}$ first collapsed, while those processed at $15{ }^{\circ} \mathrm{C}$ remained relatively stable, which resulted in the formation of a porous structure with a highest final height of $0.9 \mathrm{~mm}$. This result was because the GEL is a thermo-sensitive polymer and its viscosity increased with a decrease in the ambient temperature. Low viscosity is beneficial to processing, but not favorable for molding (Nguyen et al. 1998). For this reason, a barrel temperature of $15^{\circ} \mathrm{C}$ was utilized.
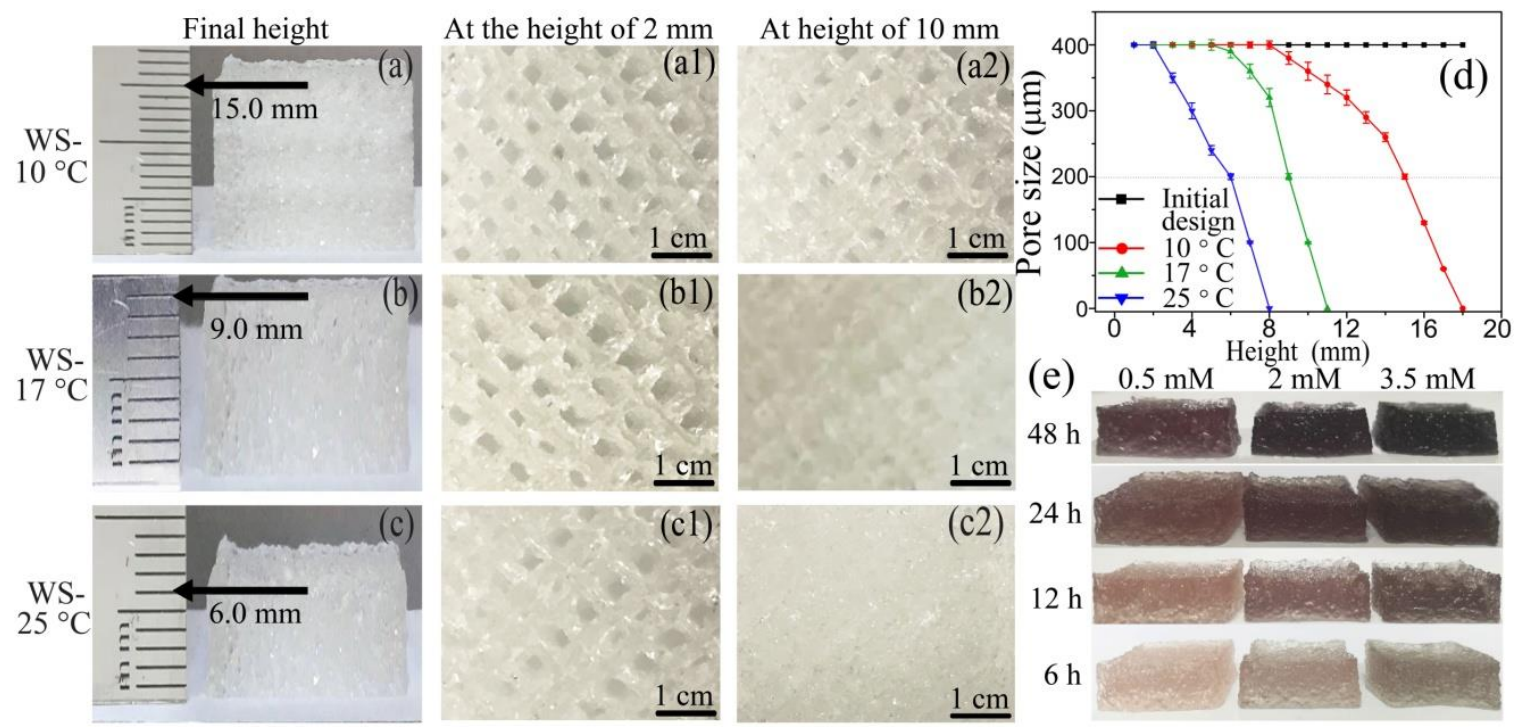

Fig. 5. Optical images of the struts printed at different work stage temperatures. The final heights for the struts printed at WS- $10^{\circ} \mathrm{C}(\mathrm{a}, \mathrm{a} 1, \mathrm{a} 2), \mathrm{WS}-17^{\circ} \mathrm{C}(\mathrm{b}, \mathrm{b} 1, \mathrm{~b} 2)$, and WS- $25^{\circ} \mathrm{C}(\mathrm{c}, \mathrm{c} 1, \mathrm{c} 2)$ were $15.0 \mathrm{~mm}, 9.0 \mathrm{~mm}, 6.0 \mathrm{~mm}$, and $6.0 \mathrm{~mm}$, respectively; The curves of the pore sizes with the increasing heights of the struts printed at different barrel temperatures $(p<0.05)$. The final strut height was the abscissa value of the intersection point of the curves and the gray dotted line at ordinate value of $200 \mu \mathrm{m}$ (d); Optical images of the 10\%-CNF/GEL-5 lattices after crosslinking treatment indicated that the color of the structures became darker and darker with an increasing genipin concentration and crosslinking time (e)

The above study of the processing temperature in the barrel facilitated the construction of better porous structures; however, the thickness of the printed strut was not still greater than $9 \mathrm{~mm}$. To obtain an optimal strut thickness, the temperature of the work stage for the 10\%-CNF/GEL-5 hydrogel was further investigated. A series of struts were printed at different work stage temperatures (WS-10 ${ }^{\circ} \mathrm{C}$, WS $-17{ }^{\circ} \mathrm{C}$, and WS-25 ${ }^{\circ} \mathrm{C}$ ) with the same barrel temperature of $15{ }^{\circ} \mathrm{C}$. According to the results of the rheological test, the $T_{\text {sol-gel }}$ for the $10 \%$-CNF/GEL-5 hydrogel was $30.1{ }^{\circ} \mathrm{C}$; therefore, these temperatures for the work stage could be used to observe the gelation temperature of the $10 \%$-CNF/GEL-5 hydrogel. Fig. 5a, 5b, and 5c show optical images of the struts printed at three different 
work stage temperatures (WS-10 ${ }^{\circ} \mathrm{C}$, WS- $17{ }^{\circ} \mathrm{C}$, and WS-25 ${ }^{\circ} \mathrm{C}$, respectively) with the same barrel temperature of $15^{\circ} \mathrm{C}$, and Fig. $5 \mathrm{~d}$ shows the changes in the pore size with the increasing strut height. As was expected, the structures had a homogeneous pore size if their thickness was within an appropriate height. However, the pores in the structure collapsed as the height increased because of the limitation of heat transfer from the working stage in the direction of the strut thickness. The final heights for the struts printed at WS$10{ }^{\circ} \mathrm{C}$, WS- $17{ }^{\circ} \mathrm{C}$, and WS-25 ${ }^{\circ} \mathrm{C}$ were $15.0 \mathrm{~mm}, 9.0 \mathrm{~mm}$, and $6.0 \mathrm{~mm}$, respectively. For this reason, a working stage temperature of $10^{\circ} \mathrm{C}$ was utilized.

Kim et al. (2016) prepared porous struts using a pure COL hydrogel through 3D printing. In their work, because of the weak mechanical properties of the pure COL, the maximal height of the printed strut could reach $4 \mathrm{~mm}$. This value was lower than our maximal of $15 \mathrm{~mm}$ in our work. That was because that the CNF as a reinforcer could interact with the GEL to form a complex triple-helix long chain structure, which improved the stability and mechanical strength of the printed strut. In addition, the CNF could be applied for other bio-inks, such as COL, fibrin, hyaluronate acid, alginate, chitosan, and agarose, to improve the stabilities of the printed constructs for 3D printing.

\section{Crosslinking Efficiency}

According to a previous report (Sundararaghavan et al. 2008), it was safe for L929 fibroblasts to be cultured in a COL-based biomaterial with a genipin concentration of approximately $1 \mathrm{mM}$ for $24 \mathrm{~h}$, while genipin concentrations of $5 \mathrm{mM}$ resulted in critical cell damage. Therefore, genipin solutions with concentrations of $0.5 \mathrm{mM}, 2 \mathrm{mM}$, and 3.5 $\mathrm{mM}$ were used to investigate the effect on the crosslinking efficiency in this study. The crosslinking efficiencies of 10\%-CNF/GEL-5 crosslinked with different concentrations of genipin for various times are shown in Table 2. It was observed that increases in both the genipin concentration and crosslinking time improved the crosslinking efficiency of the scaffolds. Additionally, the crosslinking efficiency obviously increased when the crosslinking time increased from 6 to $24 \mathrm{~h}$, but it changed little when the crosslinking time was greater than $24 \mathrm{~h}$. This finding suggested that the crosslinking reaction of genipin with GEL mainly occurs within the first $24 \mathrm{~h}$. Figure 5e shows optical images of the scaffolds crosslinked with various genipin solutions and crosslinking times. With an increasing genipin concentration and crosslinking time, the color of the crosslinked structure darkened and gradually became dark blue. Together with the crosslinking efficiency data shown in Table 2, these findings supported the conclusion that the color of the structure became deeper with an increasing crosslinking efficiency.

Table 2. Crosslinking Efficiency with Different Concentrations of Genipin at Different Crosslinking Times

\begin{tabular}{|c|c|c|c|c|}
\hline Concentration of & \multicolumn{5}{|c|}{ Crosslinking Efficiency (\%) } \\
\cline { 2 - 5 } Genipin (mM) & $6 \mathrm{~h}$ & $12 \mathrm{~h}$ & $24 \mathrm{~h}$ & $48 \mathrm{~h}$ \\
\hline 0.5 & $4.74 \pm 0.37^{\mathrm{a}}$ & $7.67 \pm 0.52^{\mathrm{a}}$ & $12.15 \pm 0.59^{\mathrm{a}}$ & $14.12 \pm 0.73^{\mathrm{a}}$ \\
\hline 2 & $10.41 \pm 0.47^{\mathrm{b}}$ & $19.91 \pm 0.81^{\mathrm{b}}$ & $30.64 \pm 1.64^{\mathrm{b}}$ & $35.46 \pm 1.82^{\mathrm{b}}$ \\
\hline 3.5 & $18.43 \pm 0.79^{\mathrm{c}}$ & $32.39 \pm 1.76^{\mathrm{c}}$ & $60.24 \pm 2.51^{\mathrm{c}}$ & $66.47 \pm 2.76^{\mathrm{c}}$ \\
\hline \multicolumn{4}{|c|}{$\begin{array}{l}\text { *Values of the crosslinking efficiency represent the mean } \pm \text { standard deviation of three } \\
\text { replicates } \\
\text { Values in a column with different superscripts are significantly different }(p<0.05)\end{array}$} \\
\hline
\end{tabular}

Jiang et al. (2018). "CNF-reinforced gelatin hydrogel," BioResources 13(3), 5909-5924. 


\section{Cell Viability and Proliferation}

The cell growth inhibition and induction of apoptosis of the G-10\%-CNF/GEL-5 hydrogel were evaluated. The $3 \mathrm{~T} 3$ cells were separately cultured with DMEM with $10 \%$ FBS and pen/strep (1\%), and the leaching liquor of the G-10\%-CNF/GEL-5 hydrogel. The above two series of tests were considered as the control and G-10\%-CNF/GEL-5 groups, respectively.

The cell growth inhibition of the G-10\%-CNF/GEL-5 hydrogel as a scaffold material for TE was evaluated by the CCK-8 assay. Figure 6a shows that the optical density (OD) values showed a gradual increasing trend with an increasing culture time, and there were no significant differences in the OD values between the G-10\%-CNF/GEL-5 and control groups. This finding suggested that the leaching liquor of the G-10\%-CNF/GEL-5 hydrogel could support the growth and proliferation of $3 \mathrm{~T} 3$ cells.

To assess the induction of apoptosis by the G-10\%-CNF/GEL-5 hydrogel, live/dead cell staining was performed according to the instructions given by the manufacturer to inspect the cell viability. Figure $6 b_{1}$ and $6 b_{2}$ show the live cells (), which are blue, and Fig. $6 b_{1}$ 'and $6 b_{2}$ ' show the dead cells, which are red. The merged images of the viable and dead cells are shown in Fig. 6b. "'and $6 b_{2}$ ". Evidently, there were many viable 3 T3 cells and few dead cells in both the G-10\%-CNF/GEL-5 and control groups. The apoptosis rates was $1.29 \pm 0.04 \%$ and $1.05 \pm 0.03 \%$ for the G-10\%-CNF/GEL-5 test group and control group, respectively ( 5 biological replicates for G-10\%-CNF/GEL-5 test group and control group. $p<0.05)$. Moreover, there were minor differences in the number of dead cells between the G-10\%-CNF/GEL and control groups, which suggested that the G-10\%-CNF/GEL-5 hydrogel does not obviously induce apoptosis above the background level.

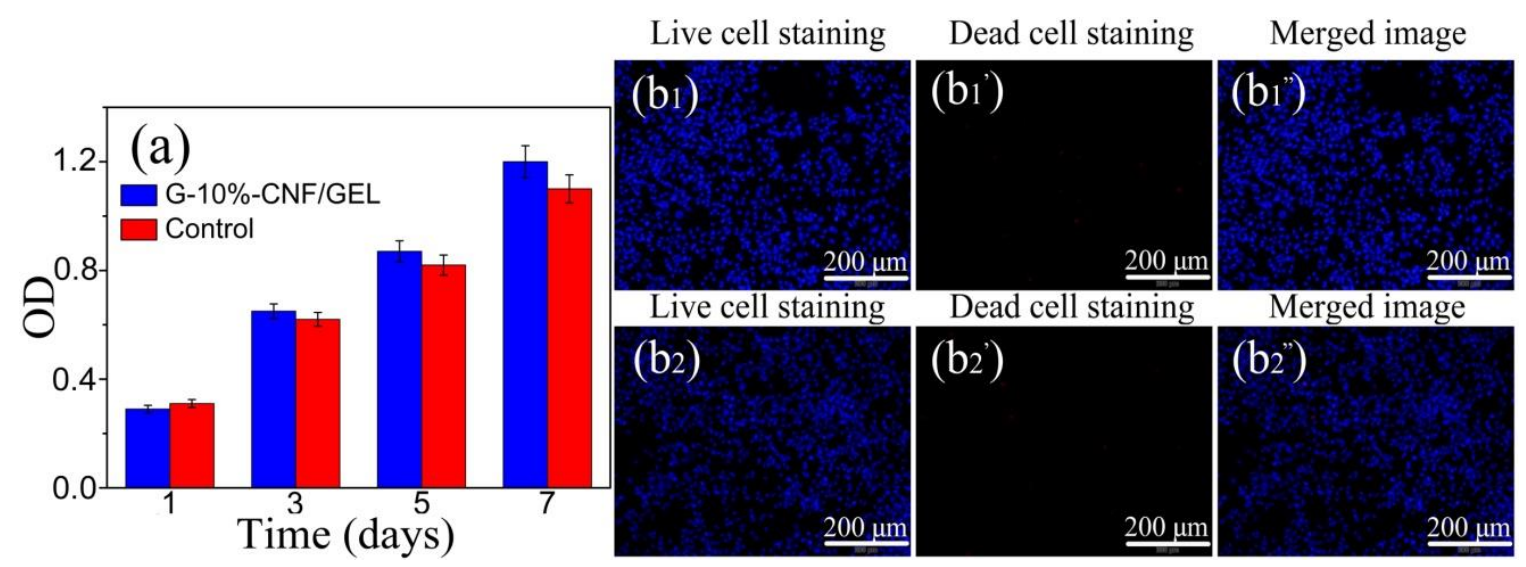

Fig. 6. Results of the cytotoxicity testing with the CCK-8 assay $(p<0.05)$ indicated that there were no significant differences in the OD values between the G-10\%-CNF/GEL-5 and control groups (a); Live/dead cell staining after culture for $48 \mathrm{~h}, \mathrm{G}-10 \%-C N F / G E L-5$ test group ( $\left.b_{1}, b_{1}{ }^{\prime}, b_{1}{ }^{\prime \prime}\right)$, control group ( $b_{2}, b_{2}{ }^{\prime}, b_{2}$ '). The apoptosis rates was $1.29 \pm 0.04 \%$ and $1.05 \pm 0.03 \%$ for the $\mathrm{G}-10 \%$ CNF/GEL-5 test group and control group, respectively $(p<0.05)$.

\section{CONCLUSIONS}

1. A new cellulose nanofibril/gelatin (CNF/GEL)-based hydrogel was proposed as a 3D printing bio-ink. The optimal hydrogel of 10\%-CNF/GEL-5 had a mechanical strength which was approximately 5.75-fold higher than that of the pure GEL. 
2. By manipulating processing parameters of 3D printing, $10 \%-C N F / G E L-5$ scaffold with good shape fidelity and interconnected porous structure was successfully obtained.

3. The CNF could be applied for other bio-inks, such as collagen (COL), fibrin, hyaluronate acid, alginate, chitosan, and agarose, to improve the stabilities of the printed constructs for 3D printing.

4. 10\%-CNF/GEL-5 composite hydrogel with good biocompatibility, which was confirmed using CCK-8 and Hoechst 33342/PI double-staining assays, has potential to be used as a 3D bio-ink for application in tissue repair.

\section{ACKNOWLEDGEMENTS}

This research was supported by the Excellent Doctoral Thesis Support Program of Yangzhou University, the top Talents Support Program of Yangzhou University, the Natural Science Foundation of China (81770018), and the City and School Cooperation Project.

\section{REFERENCES CITED}

Bäckdahl, H. (2008). Engineering the Shape of Bacterial Cellulose and Its Use as Blood Vessel Replacement, Ph.D. Dissertation, Chalmers University of Technology, Gothenburg, Sweden.

Bhattacharjee, T., Zehnder, S. M., Rowe, K. G., Jain, S., Nixon, R. M., Sawyer, W. G., and Angelini, T. E. (2015). "Writing in the granular gel medium," Science Advances 1(8). DOI: 10.1126/sciadv.1500655

Colosi, C., Shin, S. R., Manoharan, V., Massa, S., Costantini, M., Barbetta, A., Dokmeci, M. R., Dentini, M., and Khademhosseini, A. (2016). "Microfluidic bioprinting of heterogeneous 3D tissue constructs using low viscosity bioink," Adv. Mater.28(4), 677-684. DOI: 10.1002/adma.201503310

Das, S., Pati, F., Choi, Y. J., Rijal, G., Shim, J. H., Kim, S. W., Ray, A. R., Cho, D. W., and Ghosh, S. (2015). "Bioprintable, cell-laden silk fibroin-gelatin hydrogel supporting multilineage differentiation of stem cells for fabrication of threedimensional tissue constructs," Acta Biomater.11, 233-246. DOI: 10.1016/j.actbio.2014.09.023

Dawood, A., Marti, B. M., Sauret-Jackson, V., and Darwood, A. (2015). "3D printing in dentistry," Brit. Dent. J.219, 521-529. DOI: 10.1038/sj.bdj.2015.914

Dimida, S., Demitri, C., De Benedictis, V. M., Scalera, F., Gervaso, F., and Sannino, A. (2015). "Genipin-cross-linked chitosan-based hydrogels: Reaction kinetics and structure-related characteristics," J. Appl. Polym. Sci.132(28), 1-8. DOI: 10.1002/app.42256

Do, A.-V., Khorsand, B., Geary, S. M., and Salem, A. K. (2015). "3D printing of scaffolds for tissue regeneration applications," Adv. Healthc. Mater.4(12), 1742-1762. DOI: 10.1002/adhm.201500168

Gao, C., Gao, Q., Li, Y., Rahaman, M. N., Teramoto, A., and Abe, K. (2013). "in vitroevaluation of electrospun gelatin-bioactive glass hybrid scaffolds for bone 
regeneration," J. Appl. Polym. Sci.127(4), 2588-2599. DOI: 10.1002/app.37946

Hassan, M. L., Fadel, S. M., El-Wakil, N. A., and Oksman, K. (2012). "Chitosan/rice straw nanofibers nanocomposites: Preparation, mechanical, and dynamic thermomechanical properties," J. Appl. Polym. Sci.125(S2), E216-E222. DOI: 10.1002/app.36606

Highley, C. B., Rodell, C. B., and Burdick, J. A. (2015). "Direct 3D printing of shearthinning hydrogels into self-healing hydrogels," Adv. Mater.27(34), 5075-5079. DOI: 10.1002/adma.201501234

Jacobs, S., Grunert, R., Mohr, F. W., and Falk, V. (2008). "3D-imaging of cardiac structures using 3D heart models for planning in heart surgery: A preliminary study," Interact. Cardiov. Th.7(1), 6-9.

Jiang, Y., Zhou, J., Zhang, Q., Zhao, G., Heng, L., Chen, D., and Liu, D. (2017). "Preparation of cellulose nanocrystals from Humulus japonicus stem and the influence of high temperature pretreatment," Carbohyd. Polym.164, 284-293. DOI: 10.1016/j.carbpol.2017.02.021

Kim, Y. B., Lee, H., and Kim, G. H. (2016). "Strategy to achieve highly porous/ biocompatible macroscale cell blocks, using a collagen/genipin-bioink and an optimal 3D printing process," ACS Appl. Mater. Inter.8(47), 32230-32240. DOI:

10.1021/acsami.6b11669

Kim, Y. H., Kim, D.-H., Hwang, J., Kim, H.-S., Lim, G. Y., Ryoo, Z. Y., Choi, S.-U., and Lee, S. (2013). "The inclusion of fetal bovine serum in gelatin/PCL electrospun scaffolds reduces short-term osmotic stress in HEK 293 cells caused by scaffold components," J. Appl. Polym. Sci.129(6), 3273-3281. DOI: 10.1002/app.39052

Lequeux, I., Ducasse, E., Jouenne, T., and Thebault, P. (2015). "Design of an antibacterial gelatin based on a covalent protein-protein coupling," J. Appl. Polym.Sci.132(15). DOI: 10.1002/app.41825

Li, W., Guo, R., Lan, Y., Zhang, Y., Xue, W., and Zhang, Y. (2014). "Preparation and properties of cellulose nanocrystals reinforced collagen composite films," J. Biomed. Mater. Res. A 102(4), 1131-1139. DOI: 10.1002/jbm.a.34792

Lu, A., Ma, Z., Zhuo, J., Sun, G., and Zhang, G. (2013). "Layer-by-layer structured gelatin nanofiber membranes with photoinduced antibacterial functions," J. Appl. Polym. Sci.128(2), 970-975. DOI: 10.1002/app.38131

Mathew, A. P., Oksman, K., Pierron, D., and Harmand, M. F. (2013). "Biocompatible fibrous networks of cellulose nanofibres and collagen crosslinked using genipin: Potential as artificial ligament/tendons," Macromol. Biosci.13(3), 289-298. DOI: 10.1002/mabi.201200317

Müller, W. E. G., Schröder, H. C.,Neufurth, M., Tolba, E., Wang, S., Link, T., Al-Nawas, B., and Wang, X. (2015). "A new printable and durable $N, O$-carboxymethyl chitosan$\mathrm{Ca}^{2+}$-polyphosphate complex with morphogenetic activity," J. Mater. Chem. B 3(8), 1722-1730. DOI: 10.1039/C4TB01586J

Murphy, S. V., and Atala, A. (2014). "3D bioprinting of tissues and organs," Nat. Biotechnol.32(8), 773-785. DOI: 10.1038/nbt.2958

Ng, W. L., Yeong, W. Y., and Naing, M. W. (2016). "Polyelectrolyte gelatin-chitosan hydrogel optimized for 3D bioprinting in skin tissue engineering,"International Journal of Bioprinting 2(1), 53-62. DOI: 10.18063/IJB.2016.01.009

Nguyen, Q. D., Jensen, C. T. B., and Kristensen, P. G. (1998). "Experimental and modelling studies of the flow properties of maize and waxy maize starch pastes," Chem. Eng. J.70(2), 165-171. DOI: 10.1016/S0923-0467(98)00081-5 
Obregon, F., Vaquette, C., Ivanovski, S., Hutmacher, D. W., and Bertassoni, L. E. (2015). "Three-dimensional bioprinting for regenerative dentistry and craniofacial tissue engineering," J. Dent. Res.94(9 Suppl), 143S-152S. DOI: $10.1177 / 0022034515588885$

Ofner, C. M., and Bubnis, W. A. (1996). "Chemical and swelling evaluations of amino group crosslinking in gelatin and modified gelatin matrices," Pharm. Res.13(12), 1821-1827. DOI: 10.1023/A:1016029023910

Park, K. M., and Gerecht, S. (2015). "Polymeric hydrogels as artificial extracellular microenvironments for cancer research," Eur. Polym. J. 72, 507-513. DOI: 10.1016/j.eurpolymj.2015.06.030

Pereira, R. F., and Bártolo, P. J. (2015). "3D bioprinting of photocrosslinkable hydrogel constructs," J. Appl. Polym. Sci.132(48). DOI: 10.1002/app.42458

Pei, Y. (2013). Construction, Structrue and Properties of Biomedical Materials Based on Cellulose, Doctoral thesis, Wuhan University, Wuhan, ON, China.

Peresin, M. S., Habibi, Y., Zoppe, J. O., Pawlak, J. J., and Rojas, O. J. (2010). "Nanofiber composites of polyvinyl alcohol and cellulose nanocrystals: Manufacture and characterization," Biomacromolecules 11(3), 674-681. DOI: 10.1021/bm901254n

Qv, P. (2013). Cellulose Nanofibril/Poly (lactic acid) Composites and the Interfacial Compatibility, Doctoral thesis, Beijing Forestry University, Beijing, ON, China.

Skardal, A., Mack, D., Kapetanovic, E., Atala, A., Jackson, J. D., Yoo, J., and Soker, S. (2012). "Bioprinted amniotic fluid-derived stem cells accelerate healing of large skin wounds," Stem Cell. Transl. Med.1(11), 792-802. DOI: 10.5966/sctm.2012-0088

Singh, D., Singh, D., and Han, S. S. (2016). "3D printing of scaffold for cells delivery: Advances in skin tissue engineering," Polymers 8(1), 19. DOI: 10.3390/polym8010019

Sundararaghavan, H. G., Monteiro, G. A., Lapin, N. A., Chabal, Y. J., Miksan, J. R., and Shreiber, D. I. (2008). "Genipin-induced changes in collagen gels: Correlation of mechanical properties to fluorescence," J. Biomed. Mater. Res. A 87(2), 308-320. DOI: $10.1002 / \mathrm{jbm} . \mathrm{a} .31715$

Wang, W., Zhang, X., Teng, A., and Liu, A. (2017). "Mechanical reinforcement of gelatin hydrogel with nanofiber cellulose as a function of percolation concentration," Int. J. Biol. Macromol.103, 226-233. DOI: 10.1016/j.ijbiomac.2017.05.027

Wong, J. Y. (2015). "On-site 3D printing of functional custom mallet splints for Mars analogue crewmembers," Aerosp. Med. Hum. Perf.86(10), 911-914. DOI: 10.3357/AMHP.4259.2015

Zheng, X., Zhang, Q., Liu, J., Pei, Y., and Tang, K. (2016). "A unique high mechanical strength dialdehyde microfibrillated cellulose/gelatin composite hydrogel with giant network structure," RSC Adv. 6(76), 71999-72007. DOI: 10.1016/j.ijbiomac. 2017.05.027

Zheng, J., Gao, S., Li, H., and Yao, K. (2013). "Effects of reaction conditions on intercalation between gelatin and montmorillonite: Thermodynamical impact," $J$. Appl. Polym. Sci.128(1), 54-59. DOI: 10.1002/app.38129

Article submitted: March 24, 2018; Peer review completed: May 13, 2018; Revised version received and accepted: June 4, 2018; Published: June 13, 2018.

DOI: 10.15376/biores.13.3.5909-5924 